\title{
基于萠修饰树枝形聚合物的水相温敏荧光传感体系
}

\author{
秦天依 ${ }^{a, b}$ 曾毅 $*, a, b$ 陈金平 ${ }^{a}$ 于天君 ${ }^{a}$ 李嫕 $*, a, b$ \\ $\left({ }^{a}\right.$ 中国科学院理化技术研究所 光化学转换与功能材料重点实验室 北京 100190) \\ ${ }^{b}$ 中国科学院大学 北京 100049)
}

\begin{abstract}
摘要 设计合成了外围萠修饰的质子化二代聚酰胺-胺树枝形聚合物 G2 PAMAM-PyH. ${ }^{1} \mathrm{H}$ NMR 和紫外可见吸收光谱 表征确定萠基团的上载率为 $100 \%$. 吸收光谱、动态光散射(DLS)和透射电子显微镜(TEM)研究结果表明, G2 PAMAM$\mathrm{PyH}$ 在水中形成平均粒径为 $184 \mathrm{~nm}$ 双分子膜囊泡结构. 水相中 G2 PAMAM-PyH 囊泡体系呈现萠单体和激基缔合物的 双荧光发射, $1 \sim 70{ }^{\circ} \mathrm{C}$ 范围内, 随温度升高萠单体荧光逐渐增强, 而萠激基缔合物发光单调减弱, 苂光颜色由绿变蓝, 萠激基缔合物与萠单体发光比 $\left(I_{495} \mathrm{~nm} / I_{398} \mathrm{~nm}\right)$ 随温度变化具有良好的可重复性, 符合公式 $I_{495} \mathrm{~mm} / I_{398 \mathrm{~nm}}=28.23-0.68 \mathrm{t}+$ $3.21 \times 10^{-3} t^{2}+1.83 \times 10^{-5} t^{3}$, 可用于水相体系内部温度梯度的表征, 在 $1 \sim 70{ }^{\circ} \mathrm{C}$ 范围温度测量灵敏度高于 $0.9{ }^{\circ} \mathrm{C}$. 本工 作为温度苂光探针传感体系的构筑提供了一种新方法.
\end{abstract}

关键词＼cjkstart温度检测；苂光传感; 聚酰胺-胺树枝形聚合物; 芘

\section{Pyrenyl Peripheral-Decorated Polyamidoamine Dendrimer for Fluorescent Temperature Detection in Aqueous Phase}

\author{
Qin, Tianyi ${ }^{a, b} \quad$ Zeng, Yi* $^{*, a, b} \quad$ Chen, Jinping $^{a} \quad$ Yu, Tianjun ${ }^{a} \quad \mathrm{Li}, \mathrm{Yi}^{*}, a, b$ \\ ( ${ }^{a}$ Key Laboratory of Photochemical Conversion and Optoelectronic Materials, Technical Institute of Physics and Chemistry, \\ Chinese Academy of Sciences, Beijing 100190) \\ ( ${ }^{b}$ University of Chinese Academy of Sciences, Beijing 100049)
}

\begin{abstract}
A protonated polyamidoamine (PAMAM) dendrimer of generation 2 with pyrenyl attached to its periphery (G2 PAMAM-PyH) was designed and synthesized. G2 PAMAM-Py was synthesized by a condensation of the terminal amino group of the PAMAM dendrimer and the aldehyde group of 1-pyrenecarboxaldehyde followed by a reduction of Schiff base through "one pot" reaction. G2 PAMAM-Py was further protonated by adding $\mathrm{HCl}$, giving the target product G2 PAMAM$\mathrm{PyH}$. The structure of G2 PAMAM-PyH was characterized by NMR, FTIR, and MS. The functionalization extent of the peripheral amino groups of PAMAM by pyrenyl is $100 \%$ according to the ${ }^{1} \mathrm{H}$ NMR and UV-visible spectra. The amphiphilic G2 PAMAM-PyH is soluble in water with a critical aggregation concentration of $3.3 \times 10^{-7} \mathrm{~mol} \cdot \mathrm{dm}^{-3}$. Absorption, dynamic light scattering (DLS), and transmission electronic microscopy (TEM) studies demonstrate that G2 PAMAM-PyH exists as vesicle with a bilayer membrane and an average hydrodynamic diameter of $c a .184 \mathrm{~nm}$ in aqueous phase. G2 PAMAM-PyH in aqueous phase exhibits dual fluorescence, pyrenyl monomer and excimer emission. The pyrenyl monomer fluorescence increases slightly and the pyrenyl excimer emission decreases monotonically upon temperature raising from 1 to $70{ }^{\circ} \mathrm{C}$. Meanwhile, the fluorescence color changes from green (low temperature) to blue (high temperature). The monomer emission enhancement is mainly attributed to less formation of excimer when rising temperature. The fluorescence intensity ratio of pyrenyl excimer to pyrenyl monomer $\left(I_{495 \mathrm{~nm}} / I_{398 \mathrm{~nm}}\right)$ changes with varying temperature recoverably, and the relationship between $I_{495 \mathrm{~nm}} / I_{398 \mathrm{~nm}}$ and temperature can be expressed as $I_{495 \mathrm{~nm}} / I_{398 \mathrm{~nm}}=28.23-0.68 t+3.21 \times 10^{-3} t^{2}+1.83 \times 10^{-5} t^{3}$. The accuracy for the measurement of the temperature is better than $0.9{ }^{\circ} \mathrm{C}$ in the temperature range of $1 \sim 70{ }^{\circ} \mathrm{C}$, facilitating in situ gradient temperature measurement. The temperature gradient of aqueous phase in a glass tube is investigated by using G2 PAMAM-PyH, which is consistent with the detection result by using a thermocouple meter. This study provides a potential strategy for developing fluorescent temperature sensing system.

Keywords temperature detection; fluorescence sensor; polyamidoamine dendrimer; pyrene
\end{abstract}

\section{1 引言}

温度是基本物理量之一, 对温度的原位测量无论在 科学研究还是工业应用中都有重要意义 ${ }^{[1]}$. 常用的温度
计是基于某一温度依赖的物理性质设计，例如体积、电 导或电阻等等 ${ }^{[2]}$. 原位温度表征技术因其对分析体系干 扰性小在分析、诊断、工业、军工等领域表现出越来越

*E-mail: zengyi@mail.ipc.ac.cn; yili@mail.ipc.ac.cn

Received October 13, 2016; published October 27, 2016.

Supporting information for this article is available free of charge via the Internet at http://sioc-journal.cn.

Project supported by the National Natural Science Foundation of China (No. 21233011) and 973 Program (2013CB834703 and 2013CB834505).

项目受国家自然科学基金(No. 21233011)和 973 项目(2013CB834703 and 2013CB834505)资助. 
明显的优势 ${ }^{[3]}$, 红外、液晶等原位测温技术即是应这些 需求发展出的光学温度计 ${ }^{[4]}$. 近年来, 荧光温度传感技 术因其灵敏度高、空间分辨率高、响应时间短、可视化 效果好等优点被关注 ${ }^{[5]}$, 研究者们致力于发展新型苂光 温度探针分子和传感体系, 以适用于各类微环境及生物 体内的原位温度测量 ${ }^{[6]}$.

荧光温度探针分子工作原理通常是基于辐射跃迁 和非辐射跃迁速率随温度变化引起的苂光强度的改变, 但基于单一荧光发射强度变化的温度传感容易受到其 他因素的影响, 例如样品分布不均、激发光功率不同等 都会导致苂光强度产生波动 ${ }^{[7]}$. 为了实现温度的精确测 量, 具有双发射的比率苂光传感体系成为新的发展趋 势, 相比单纯依赖荧光强度变化的单荧光发射体系, 双 发射比率苂光探针体系具有自校准的优势, 不受探针分 子分布和激发光强度等因素的影响, 具有高的温度分辨 率 ${ }^{[8]}$; 同时双发射比率苂光探针体系还伴随有体系苂光 颜色的改变, 可用于裸眼识别体系温度的变化 ${ }^{[6 \mathrm{a}, 6 \mathrm{~d}, 6 \mathrm{e}, 9]}$.

聚酰胺一胺树枝形聚合物(PAMAM) 分子链段由大 量酰胺键和叔胺组成, 分子骨架本身具有一定的水溶 性, 外围大量的胺基可用于功能化修饰 ${ }^{[10]}$. 我们曾通过 外围不同官能团修饰得到了具有光捕获、苂光传感和控 制释放等性质的聚酰胺-胺树枝形聚合物 ${ }^{[11]}$, 在以䒺基 才修饰的 PAMAM 体系中观察到紫外区域萗单体和激 基缔合物双苂光发射 ${ }^{[12]}$. 如果将外围的荎基团替换为 具有更大共轭体系的萠基团, 将可得到一个发光在可见 光区的双荧光发射体系, 有可能利用萠单体和激基缔合 物发光对温度响应程度的不同, 将其发展成为一个新型 温度响应比率苂光探针体系 ${ }^{[13]}$.

本工作设计合成了外围萠基团修饰的二代聚酰胺胺树枝形聚合物 G2 PAMAM-Py, 通过盐酸质子化得到 水中溶解性更好的质子化产物 G2 PAMAM-PyH. G2 PAMAM-PyH 在水中形成双分子膜囊泡, 呈现萠单体和 激基缔合物的双苂光发射. $1 \sim 70{ }^{\circ} \mathrm{C}$ 范围内, 随温度增 加萠单体荧光小幅增加, 而萠激基缔合物荧光单调下 降, 利用萠激基缔合物和萠单体苂光强度比随温度改变 的规律变化, G2 PAMAM-PyH 可作为温度探针表征水 相体系的温度梯度变化. 本工作为温度荧光探针传感体 系的构筑提供了新的实验依据.

\section{2 结果与讨论}

\section{1 化合物的合成}

PAMAM 骨架采用发散法合成, 十二烷基二胺和丙 烯酸甲酯的 Michael 加成得到末端为丙烯酸甲酯的 0.5 代产物 G0.5 PAMAM, G0.5 PAMAM 用乙二胺对酯键进 行氨解, 得到末端为伯胺的整代数产物; 重复这两步反 应即可得到 2 代产物 G2 PAMAM, 具体步骤参考文 献 ${ }^{[11 a]}$. 1-萠甲醛参考文献报道方法合成 ${ }^{[14]}$. 外围萠基团
修饰的聚酰胺一胺树枝形聚合物 G2 PAMAM-Py 的合成 路线如图 1 所示, G2 PAMAM 分子外围的伯胺和 1-萠甲 醛发生席夫碱缩合反应，再经碀氢化钠还原 “一锅” 反 应得到粗产物，粗产物经聚苯乙烯凝胶色谱柱层析分离 纯化，外围萠基团上载率 $100 \%$ 的产物(根据 ${ }^{1} \mathrm{H}$ NMR 和 吸收光谱结果)G2 PAMAM-Py 产率为 $33 \%$. 为了增加 G2 PAMAM-Py 在水中的溶解度, 将 G2 PAMAM-Py 溶 于少量四氢呋喃中, 滴加过量盐酸进行质子化, 析出沉 淀即为质子化后的产物 G2 PAMAM-PyH. 模型化合物 (Model)及其质子化产物(ModelH)由 1-萠甲醛和 1-丙胺 按照类似方法制备得到(图 1). 所有化合物均通过了红 外光谱、核磁共振谱和质谱的鉴定(图 $\mathrm{S} 1 \sim \mathrm{S} 14$ ), G2 PAMAM-Py 和 Model 溶于四氢呋喃(THF)和氯仿等有机 溶剂, 质子化后的 G2 PAMAM-PyH 和 ModelH 不溶于 有机溶剂, 但能溶于水.

\section{2 稳态光谱及聚集体表征}

测试了 ModelH 和 G2 PAMAM-PyH 在水中的紫外 吸收光谱, 如图 2(a)所示. 水溶液中 ModelH 和 G2 PAMAM-PyH 基本呈萠吸收光谱的特征，但相同萠基团 浓度下 $\left([\mathrm{Py}]=1.0 \times 10^{-5} \mathrm{~mol} \cdot \mathrm{dm}^{-3}\right) \mathrm{G} 2$ PAMAM-PyH 的 吸光度相比 ModelH 大大降低, 并伴有轻微的变宽和红 移(约 $6 \mathrm{~nm}$ ). 为了确认 G2 PAMAM-PyH 吸收的降低不 是由于底物析出所致，进一步测定了不同浓度 G2 PAMAM-PyH 在水中的吸收光谱(图 $\mathrm{S} 15$ ), 在 $[\mathrm{Py}]=2.0$ $\times 10^{-6} \sim 8.9 \times 10^{-5} \mathrm{~mol} \cdot \mathrm{dm}^{-3}$ 范围内，萠基团吸光度随 其浓度增加而线性增加, 排除了 G2 PAMAM-PyH 相比 ModelH 吸光度降低是底物析出所致的原因, 推测 G2 PAMAM-PyH 吸收的降低是因为其在水中形成了聚集 体，萠基团在聚集体中的吸收降低并红移.

进一步测定了 ModelH 和 G2 PAMAM-PyH 在水中 的荧光发射光谱 $\left([\mathrm{Py}]=1 \times 10^{-5} \mathrm{~mol} \cdot \mathrm{dm}^{-3}\right)$. 图 2(b)为对 激发波长 $350 \mathrm{~nm}$ 处吸收归一化后的苂光光谱, 发光范 围在 368 428 nm, 而 G2 PAMAM-PyH 只能观察到很 弱的萠单体发光，但在长波长方向观察到很强的萠激基 缔合物的发光，最大发射峰在 $495 \mathrm{~nm}$ 处. 进一步浓度稀 释实验表明 G2 PAMAM-PyH 在水中形成的激基缔合 物有分子间的成分, 说明 G2 PAMAM-PyH 在水中应形 成了聚集体. 测定了不同浓度下 G2 PAMAM-PyH 水溶 液的发光光谱, 将 $495 \mathrm{~nm}$ 处的萠激基缔合物荧光强度与 $398 \mathrm{~nm}$ 处萠单体苂光强度的比值 $\left(I_{495} \mathrm{~nm} / I_{398 \mathrm{~nm}}\right)$ 对 $\mathrm{G} 2$ PAMAM-PyH 浓度作图, 得到如图 2(b)插入图所示曲线. 曲线明显呈两部分, 低浓度时比率苂光随浓度降低大幅 减小，在较高浓度区域比率苂光随浓度增加线性增加， 显然 G2 PAMAM-PyH 在拐点浓度以上发生了聚集，从 低浓度和高浓度区域线性拟合线的交叉点得到临界聚 集浓度 $(\mathrm{CAC})$ 为 $3.3 \times 10^{-7} \mathrm{~mol} \cdot \mathrm{dm}^{-3}\left([\mathrm{Py}]=5.3 \times 10^{-6}\right.$ $\left.\mathrm{mol} \cdot \mathrm{dm}^{-3}\right)$. 

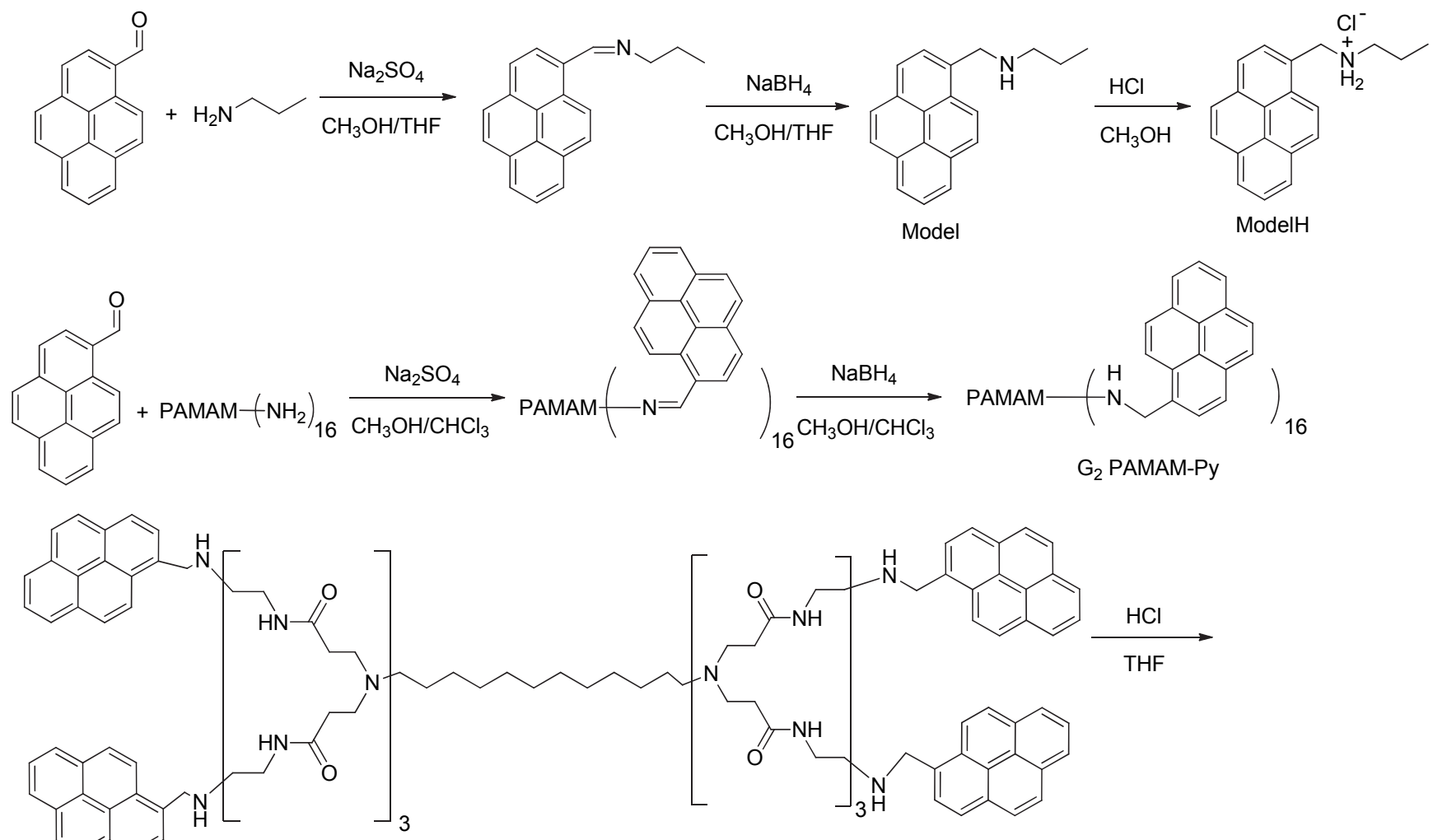

$\mathrm{G}_{2}$ PAMAM-Py

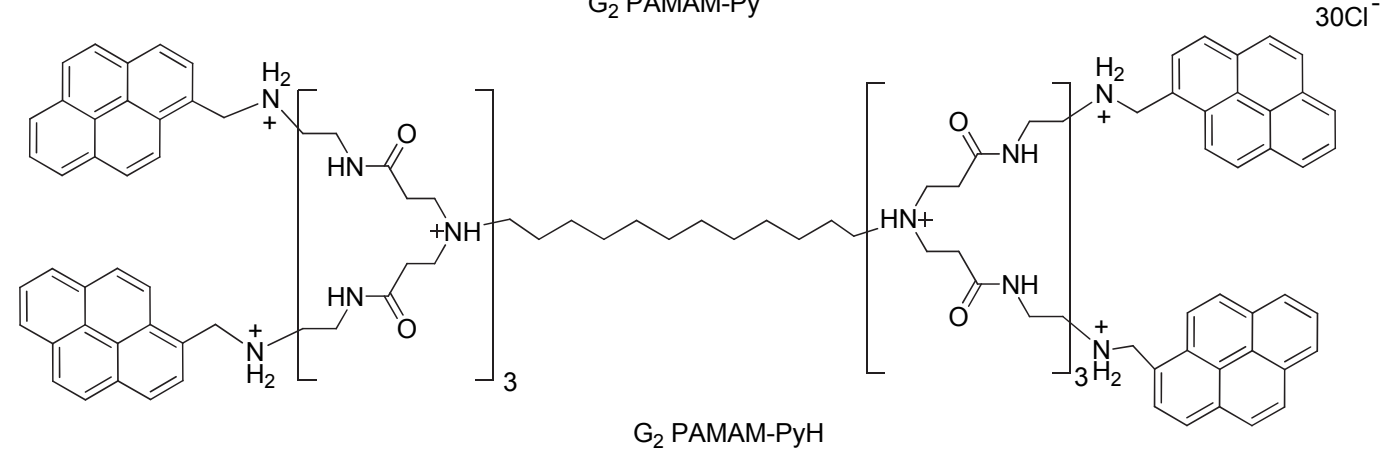

图 1 ModelH 和 G2 PAMAM-PyH 的合成路线

Figure 1 Synthetic routes of ModelH and G2 PAMAM-PyH
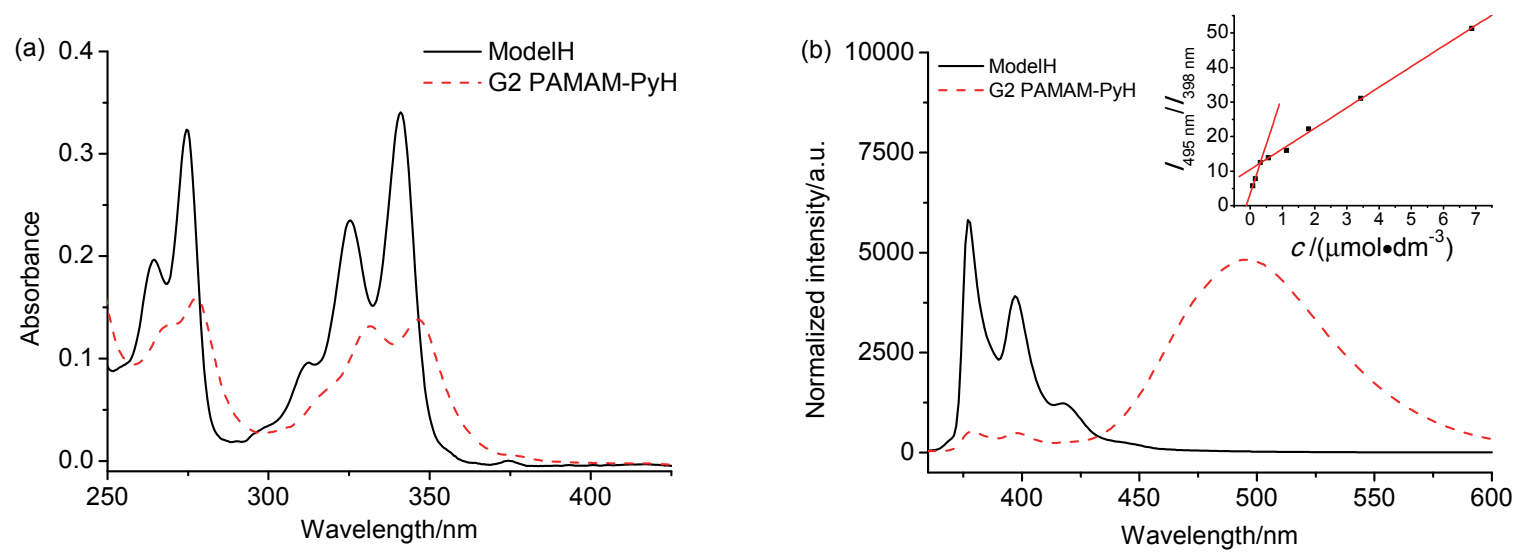

图 2 (a) ModelH(实线)和 G2 PAMAM-PyH(虚线)在水中的吸收光谱. (b) 相同官能团浓度下 ModelH 和 G2 PAMAM-PyH 归一化苂光光谱 $\left(\lambda_{\mathrm{ex}}=350\right.$ $\left.\mathrm{nm},[\mathrm{Py}]=1.0 \times 10^{-5} \mathrm{~mol} \cdot \mathrm{dm}^{-3}\right)$; 插入图为比率苂光 $\left(I_{495} \mathrm{~nm} / I_{398} \mathrm{~nm}\right)$ 随 $\mathrm{G} 2 \mathrm{PAMAM}-\mathrm{PyH}$ 浓度的变化(点)拟合曲线(实线)

Figure 2 Absorption (a) and normalized emission (b) spectra of ModelH (solid line) and G2 PAMAM-PyH (dash line) in $\mathrm{H}_{2} \mathrm{O}\left(\lambda_{\mathrm{ex}}=350 \mathrm{~nm}\right.$, [Py]= $\left.1.0 \times 10^{-5} \mathrm{~mol} \cdot \mathrm{dm}^{-3}\right)$. Inset: Ratio fluorescence intensity $\left(I_{495 \mathrm{~nm}} / I_{398} \mathrm{~nm}\right)$ as a function of the concentration of G2 PAMAM-PyH 
动态光散射(Dynamic Light Scattering, DLS)实验进 一步证实 G2 PAMAM-PyH 在水中呈聚集状态. DLS 实 验表明(图 S16), G2 PAMAM-PyH 在水溶液中的平均粒 径为 $184 \mathrm{~nm}$, 说明其以聚集体形式存在. 为了解 $\mathrm{G} 2$ PAMAM-PyH 在水中形成聚集体的详细信息, 利用透射 电子显微镜(TEM)对其进行了表征, 图 3 为 TEM 测试结 果. G2 PAMAM-PyH 在水中聚集后呈球形或椭球形结 构, 外围和中间部分有明显的反差, 其外壁厚约为 5 $\mathrm{nm}$, 约为 G2 PAMAM-PyH 分子尺寸的两倍, 显然 $\mathrm{G} 2$ PAMAM-PyH 在水中形成了具有双分子膜结构的囊泡, TEM 观察到的囊泡尺寸与 DLS 测试结果一致.

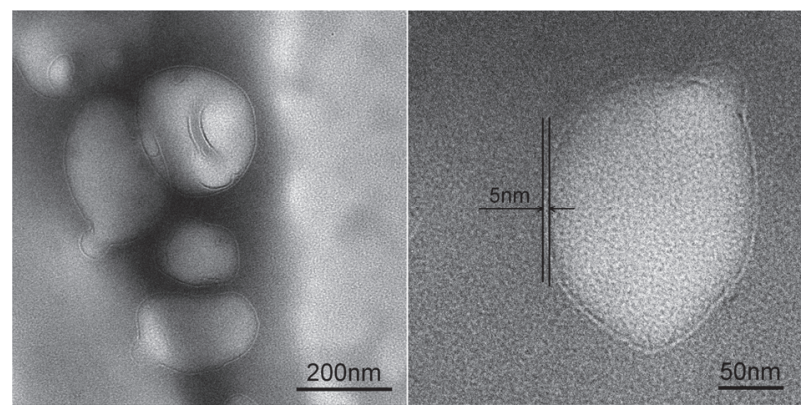

图 3 冻干水相 G2 PAMAM-PyH 体系 TEM 照片, 右图中给出壁厚为 $5 \mathrm{~nm}$

Figure 3 TEM images of G2 PAMAM-PyH vesicles with a $5 \mathrm{~nm}$ thick wall

\subsection{G2 PAMAM-PyH 比率荧光的温度响应}

温度对激基缔合物的生成有重要影响, 温度升高不 利于激基缔合物的生成, 因此, 有可能通过 G2 PAMAM$\mathrm{PyH}$ 囊泡体系中激基缔合物与单体发光比例的变化表 征体系的温度. 测定了不同温度下水相 G2 PAMAM$\mathrm{PyH}$ 囊泡体系 $\left([\mathrm{Py}]=2 \times 10^{-5} \mathrm{~mol} \cdot \mathrm{dm}^{-3}\right)$ 的苂光发射光 谱, 如图 4(a)所示, 为便于观察, 图中萠单体的发光 (365 426 nm) 放大了四倍. 升温过程 $\left(1 \sim 70{ }^{\circ} \mathrm{C}\right.$, 箭头 方向)萠单体荧光有小幅增强，而激基缔合物的发光大 幅降低. 升高温度单体和激基缔合物的非辐射跃迁均增 强, 但萠单体苂光随温度增加而增强, 其原因可归结为 升温在一定程度上抑制了激基缔合物的生成. 将 495 $\mathrm{nm}$ 处的萠激基缔合物荧光强度与 $398 \mathrm{~nm}$ 处萠单体苂光 强度的比值 $\left(I_{495} \mathrm{~nm} / I_{398 \mathrm{~nm}}\right)$ 对温度 $(t)$ 作图, 得到如图 4(b) 所示的曲线, 在 $1 \sim 70{ }^{\circ} \mathrm{C}$ 范围内, 该曲线可用多项式 $I_{495 \mathrm{~nm}} / I_{398 \mathrm{~nm}}=28.23-0.68 t+3.21 \times 10^{-3} t^{2}+1.83 \times 10^{-5} t^{3}$ (公式 1)进行拟合[拟合曲线见图 4(b)中红色实线], 该多 项式仅仅是比率苂光 $\left(I_{495} \mathrm{~nm} / I_{398} \mathrm{~nm}\right)$ 与温度 $t$ 间的数学表 达, 并无明确的物理意义, 不同温度下曲线斜率代表该 温度下测温灵敏度 $\left({ }^{\circ} \mathrm{C}^{-1}\right)^{[6 \mathrm{e}]}$. 据此, 我们可以通过测量 体系的比率荧光大小表征体系的温度. 重复测量各温度 下苂光光谱得到比率荧光标准偏差, 比率苂光标准偏差 与拟合曲线中 $\mathrm{d} I / \mathrm{d} t$ 的比值即为该温度下的测温灵敏度, 计算得到 $1{ }^{\circ} \mathrm{C}$ 和 $70{ }^{\circ} \mathrm{C}$ 的测温灵敏度分别为 $0.8{ }^{\circ} \mathrm{C}$ 和
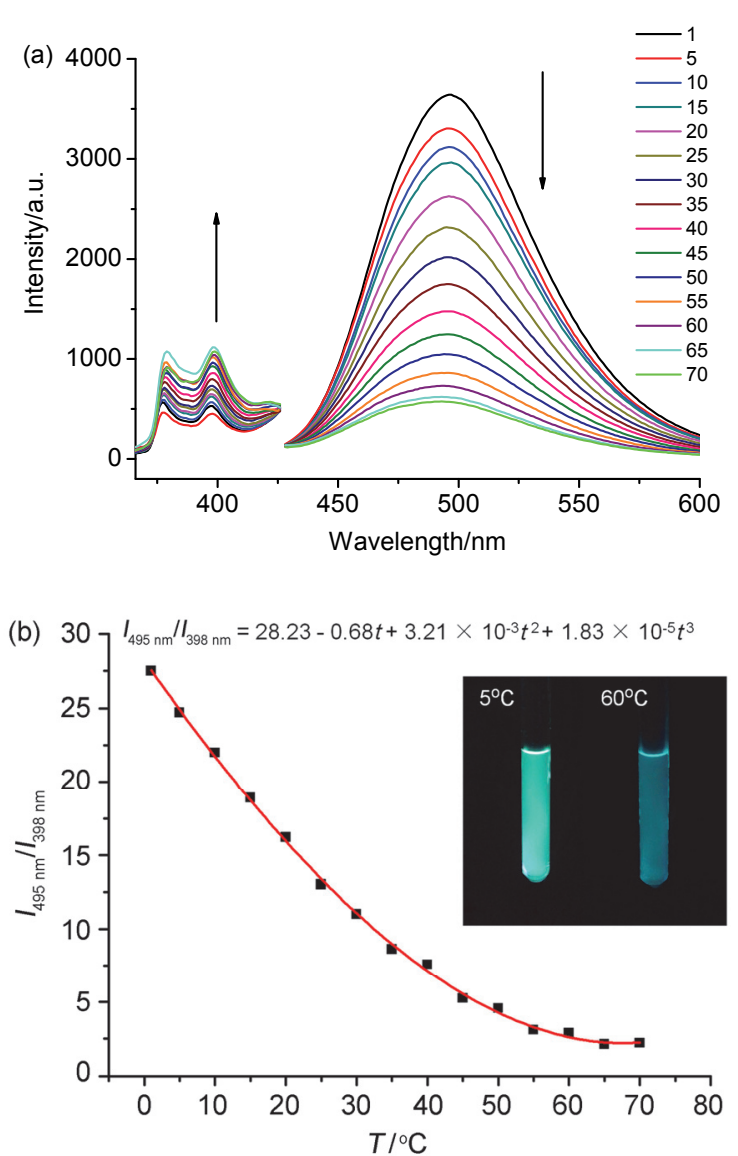

图 4 (a) G2 PAMAM-PyH 在水中的变温荧光光谱 $\left(\lambda_{\mathrm{ex}}=350 \mathrm{~nm}\right.$, [Py] $\left.=2.0 \times 10^{-5} \mathrm{~mol} \cdot \mathrm{dm}^{-3}\right), 365 \sim 426 \mathrm{~nm}$ 为放大 4 倍的光谱曲线, 箭头所 指方向为升温过程. (b) 比率苂光 $\left(I_{495} \mathrm{~nm} / I_{398} \mathrm{~nm}\right)$ 随温度的变化, 黑色点 为实验数据, 红线为拟合曲线. 插入图为 $5{ }^{\circ} \mathrm{C}$ 和 $60{ }^{\circ} \mathrm{C}$ 时 $365 \mathrm{~nm}$ 光激 发下体系的照片

Figure 4 (a) Emission spectra of G2 PAMAM-PyH in $\mathrm{H}_{2} \mathrm{O}$ at different temperatures $\left(\lambda_{\mathrm{ex}}=350 \mathrm{~nm},[\mathrm{Py}]=2.0 \times 10^{-5} \mathrm{~mol} \cdot \mathrm{dm}^{-3}\right)$, the emission spectra in $365 \sim 426 \mathrm{~nm}$ are enlarged 4 times. The arrow indicates the heating process. (b) Temperature dependence of the ratio of fluorescence intensity $\left(I_{495} \mathrm{~nm} / I_{398} \mathrm{~nm}\right)$. Inset: Fluorescent images of the G2 PAMAM$\mathrm{PyH}$ aqueous phase under $350 \mathrm{~nm}$ excitation at $5{ }^{\circ} \mathrm{C}$ and $60{ }^{\circ} \mathrm{C}$

$0.9{ }^{\circ} \mathrm{C}$, 说明在 $1 \sim 70{ }^{\circ} \mathrm{C}$ 温度范围内水相 G2 PAMAM$\mathrm{PyH}$ 囊泡体系的测温灵敏度可达 $0.9{ }^{\circ} \mathrm{C}$ 以上. 图 4(b)插 入图为 $5{ }^{\circ} \mathrm{C}$ 和 $60{ }^{\circ} \mathrm{C}$ 时水相 G2 PAMAM-PyH 囊泡体系 的苂光颜色，随着温度升高苂光发生从绿色到蓝色的显 著转变, 表明水相 G2 PAMAM-PyH 囊泡体系的荧光温 度响应具有可视化效果. 在 $5{ }^{\circ} \mathrm{C}$ 和 $60{ }^{\circ} \mathrm{C}$ 多次循环升降 温, 体系的比率苂光值 $I_{495 \mathrm{~nm}} / I_{398 \mathrm{~nm}}$ 具有良好的可回复 性(图 5), 说明水相 G2 PAMAM-PyH 囊泡体系在测试温 度范围内具有良好的稳定性, 有可能作为新型温度传感 材料应用于温度测量.

为了证明水相 G2 PAMAM-PyH 囊泡体系可应用于 液体内部温度梯度的测量, 我们将水相 G2 PAMAM$\mathrm{PyH}$ 囊泡体系置于玻璃管中，上端和下端分别加热和用 冰水冷却, 如图 6 所示. 裸眼即可观察到自上而下荧光 


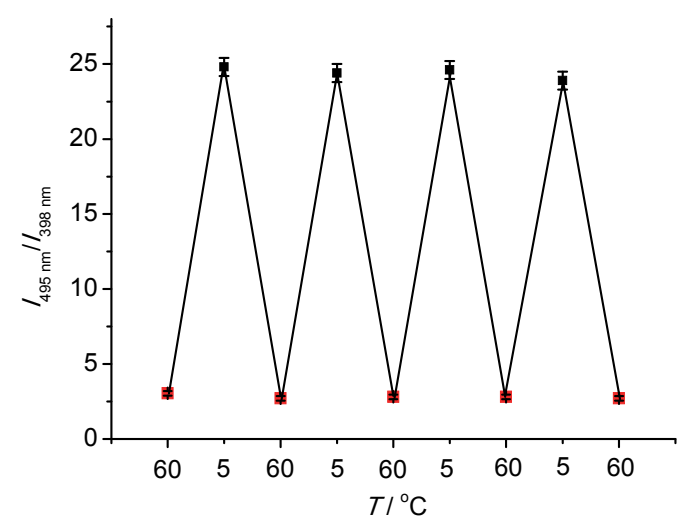

图 5 多次升降温循环水相 G2 PAMAM-PyH 囊泡体系比率荧光 Figure 5 Reversibility of ratio fluorescence intensity of G2 PAMAM-PyH aqueous dispersion at $5{ }^{\circ} \mathrm{C}$ and $60{ }^{\circ} \mathrm{C}$

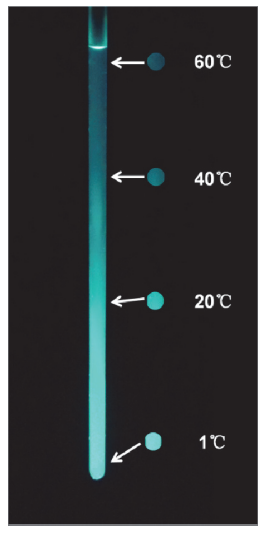

图 6 玻璃管中水相 G2 PAMAM-PyH 囊泡体系温度梯度苂光分布图 Figure 6 Photograph of fluorescence at different temperatures and the gradient fluorescence of G2 PAMAM-PyH solution in a glass tube

颜色和强度发生了明显的变化, 从高温到低温苂光颜色 从蓝色转变为绿色, 苂光强度逐渐增强. 用热电偶测得 管壁 60.0、40.2、20.1、1.1 ${ }^{\circ} \mathrm{C}$ 的位置, 利用光纤光谱仪 测得这四个温度位置的比率苂光值 $\left(I_{495} \mathrm{~nm} / I_{398} \mathrm{~nm}\right)$ 分别为 2.64、7.10、15.91 和 27.57 , 从公式(1)计算得到四个位 置相应的温度分别为 59.7、40.0、20.1、1.0 ${ }^{\circ} \mathrm{C}$, 在误差 范围内与热电偶测量结果完全一致, 证实此温度响应囊 泡可应用于水相温度梯度检测.

\section{3 结论}

本文设计合成了外围萠基团修饰的二代质子化聚 酰胺一胺树枝形聚合物 G2 PAMAM-PyH, 研究了 G2 PAMAM-PyH 在水相中的分散性质及其荧光光谱的温 度响应. 结果表明, G2 PAMAM-PyH 在水中形成平均粒 径 $184 \mathrm{~nm}$ 的双分子层囊泡结构, 水相中 G2 PAMAM$\mathrm{PyH}$ 囊泡体系呈现萠单体和激基缔合物的双苂光发射, 1 70 ${ }^{\circ} \mathrm{C}$ 范围内, 随温度升高萠单体荧光逐渐增强, 而 萠激基缔合物发光单调减弱, 萠激基缔合物与萠单体发 光比 $I_{495 \mathrm{~nm}} / I_{398 \mathrm{~nm}}$ 随温度规律变化, 具有良好的可重复
性，可用于水相温度梯度的表征，在 $1 \sim 70{ }^{\circ} \mathrm{C}$ 范围温度 测量灵敏度高于 $0.9{ }^{\circ} \mathrm{C}$. 本工作为温度响应荧光体系的 构建提供了重要参考.

\section{4 实验部分}

\section{1 试剂与仪器}

实验使用的试剂均从 $\mathrm{J} \& \mathrm{~K}$, Acros 等公司购买, 除特 别说明外所有药品均直接使用. 核磁共振 ${ }^{1} \mathrm{H}$ NMR (400 $\mathrm{MHz})$ 和 ${ }^{13} \mathrm{C}$ NMR(100 MHz)使用 Bruker Avance P-400 核磁共振仪. 质谱测试使用 Burker BIFLEX III 型飞行时 间质谱仪(MALDI-TOF), GCT-Premier (HR EI-TOF), QExactive (ESI-TOF), 9.4T Solarix 型傅立叶变换离子回旋 共振质谱仪(HR ESI-TOF). 红外光谱使用 Excalibur 3100 IR 红外光谱仪. 紫外吸收光谱使用岛津 UV-1601PC 型紫外可见吸收光谱仪. 稳态荧光光谱使 用日立 F-4600 型荧光光谱仪. 控温实验使用 Huber Ministat 230-CC 冷热恒温仪. 动态光散射测试使用 Malvern Zetasizer Nano ZS 动态光散射仪. 透射电镜使 用 JEM-1011 透射电子显微镜. 光纤光谱仪使用 Ava-2048TEC 发光光谱仪.

\section{2 化合物合成及表征}

Model 在 $100 \mathrm{~mL}$ 三口瓶中加入 $1 \mathrm{~g}$ 1-萠甲醛和 $0.36 \mathrm{~mL}$ 正丙胺, 加入 $1.2 \mathrm{~g}$ 无水硫酸钠, $5 \mathrm{~mL} \mathrm{CH}_{3} \mathrm{OH}$ 和 $5 \mathrm{~mL} \mathrm{THF}, \mathrm{N}_{2}$ 保护下加热至 $45{ }^{\circ} \mathrm{C}$ 反应 $5 \mathrm{~h}$. 冷却至室温 后加入 $0.48 \mathrm{~g}$ 嗍氢化钠, 反应 $3 \mathrm{~h}$ 后过滤，将滤液旋干后 溶于二氯甲烷，用饱和食盐水洗涤三次，有机相用无水 硫酸钠干燥后减压除去溶剂, 粗产物使用硅胶柱层析分 离, 洗脱剂为 $V\left(\mathrm{CH}_{2} \mathrm{Cl}_{2}\right) / V\left(\mathrm{CH}_{3} \mathrm{OH}\right)=20: 1$, 得 $0.97 \mathrm{~g}$ 淡黄色固体, 产率 $83 \%$; m.p. $66.2 \sim 67.2{ }^{\circ} \mathrm{C}$; UV-vis (THF) $\lambda_{\max }: 329,346 \mathrm{~nm} ;{ }^{1} \mathrm{H}$ NMR $\left(400 \mathrm{MHz}, \mathrm{CDCl}_{3}\right) \delta$ : $8.38(\mathrm{~d}, J=9.2 \mathrm{~Hz}, 1 \mathrm{H}), 8.20 \sim 8.13(\mathrm{~m}, 4 \mathrm{H}), 8.06 \sim 7.98$ (m, 4H), $4.50(\mathrm{~s}, 2 \mathrm{H}), 2.78(\mathrm{t}, J=7.2 \mathrm{~Hz}, 2 \mathrm{H}), 1.62(\mathrm{dd}$, $J=14.6,7.3 \mathrm{~Hz}, 2 \mathrm{H}), 0.95$ (t, $J=7.4 \mathrm{~Hz}, 3 \mathrm{H})$; IR (KBr) $v$ : $3424,3029,2953,2872,2817,1645,1585,1435,1137$, $845,796,709 \mathrm{~cm}^{-1}$; HRMS (EI-TOF) calcd for $\mathrm{C}_{20} \mathrm{H}_{19} \mathrm{~N}$ 273.1517, found 273.1517.

G2 PAMAM-Py 在 $100 \mathrm{~mL}$ 三口瓶中加入 G2 PAMAM $0.39 \mathrm{~g}$ 和 1-萠甲醛 $1.68 \mathrm{~g}$, 其中 1-萠甲醛与 $\mathrm{G} 2$ PAMAM 外围胺基物质的量比为 $4: 1$, 加入 $2.1 \mathrm{~g}$ 无水 硫酸钠, 溶剂为三氯甲烷和甲醇各 $5 \mathrm{~mL}$, 油浴升温至 $40{ }^{\circ} \mathrm{C}, \mathrm{N}_{2}$ 保护下搅拌反应 $24 \mathrm{~h}$. 待反应液冷却到室温后 加入嗍氢化钠 $2.2 \mathrm{~g}$, 室温反应 $3 \mathrm{~h}$.

反应结束后减压过滤除去白色固体, 有机相用饱和 食盐水洗涤三次, 用无水硫酸钠干燥后减压除去溶剂, 粗产物用聚苯乙烯凝胶色谱柱(GPC)分离, 得到产物为 淡黄色固体. 其中上载率 $100 \%$ 的产物 $200 \mathrm{mg}$, 产率 $33 \%$; UV-vis (THF) $\lambda_{\max }: 329,346 \mathrm{~nm} ;{ }^{1} \mathrm{H}$ NMR (400 
$\left.\mathrm{MHz}, \mathrm{CDCl}_{3}\right) \delta: 8.08 \sim 8.01$ (br m, 48H), $7.93 \sim 7.83(\mathrm{br}$ m, 80H), 7.74 (br d, $J=7.2 \mathrm{~Hz}, 16 \mathrm{H}$ ), 4.18 (br s, 32H), 3.14, 2.98 (br s, 56H), 2.63 (br s, 32H), 2.36 (br s, 56H), 2.18 (br s, $84 \mathrm{H}$ ), 2.01 (br s, 16H), $1.44 \sim 1.05$ (br s, $20 \mathrm{H}$ ); IR (KBr) v: 3424, 3293, 3045, 2928, 2853, 1639, 1540, 1434, 1131, 845, $729 \mathrm{~cm}^{-1}$; MS (MALDI-TOF) calcd for $\mathrm{C}_{424} \mathrm{H}_{468} \mathrm{O}_{28} \mathrm{~N}_{58} 6824.8$, found $6849.0\left(\mathrm{M}+\mathrm{Na}^{+}\right.$).

ModelH 将模型化合物 $0.3 \mathrm{~g}$ 溶于 $2 \mathrm{~mL}$ 甲醇, 加入 2 滴浓盐酸, 搅拌均匀后滴入过量乙醚中析出沉淀, 真 空干燥后得到 $0.25 \mathrm{~g}$ 淡黄色固体, 产率 75\%; m.p. $181.0 \sim 183.0{ }^{\circ} \mathrm{C}$; UV-vis $\left(\mathrm{H}_{2} \mathrm{O}\right) \lambda_{\max }$ : $325,341 \mathrm{~nm}$; ${ }^{1} \mathrm{H}$ NMR (400 MHz, $\left.\mathrm{D}_{2} \mathrm{O}\right) \delta: 8.09$ (dd, $\left.J=17.2,7.4 \mathrm{~Hz}, 2 \mathrm{H}\right)$, $7.98(\mathrm{~d}, J=7.2 \mathrm{~Hz}, 1 \mathrm{H}), 7.89$ (dd, $J=8.9,2.9 \mathrm{~Hz}, 1 \mathrm{H})$, $7.83 \sim 7.73(\mathrm{~m}, 3 \mathrm{H}), 7.60(\mathrm{t}, J=9.3 \mathrm{~Hz}, 2 \mathrm{H}), 4.42(\mathrm{~s}, 2 \mathrm{H})$, 2.98 (t, $J=8 \mathrm{~Hz}, 2 \mathrm{H}), 1.64$ (dd, $J=15.3,7.6 \mathrm{~Hz}, 2 \mathrm{H}), 0.89$ (t, $J=7.4 \mathrm{~Hz}, 3 \mathrm{H})$; IR (KBr) v: 3449, 3381, 2953, 2779, 2689, 2562, 1899, 1750, 1583, 1434, 1131, $833 \mathrm{~cm}^{-1}$; HRMS (ESI-TOF) calcd for $\mathrm{C}_{20} \mathrm{H}_{20} \mathrm{~N}$ 274.1596, found 274.1588 .

G2 PAMAM-PyH 合成方法与 ModelH 相同, 溶剂 使用四氢呋喃, 加入过量盐酸析出沉淀, 真空干燥后得 橙黄色固体，产率 75\%; UV-vis $\left(\mathrm{H}_{2} \mathrm{O}\right) \lambda_{\max }$ : $331,346 \mathrm{~nm}$; ${ }^{1} \mathrm{H}$ NMR $\left(400 \mathrm{MHz}, \mathrm{D}_{2} \mathrm{O}\right) \delta: 7.46 \sim 6.87$ (br m, 144H), 4.34 (br s, 32H), 3.34, 3.09, 2.84 (br s, 168H), 2.49 (br s, $60 \mathrm{H}$ ), $1.45 \sim 0.65$ (br m, 20H); IR (KBr) v: 3445, 3276, 3044, 2921, 2774, 2640, 2104, 1655, 1563, 1397, 1114, $834 \mathrm{~cm}^{-1}$; HRMS (ESI-TOF) calcd for $\mathrm{C}_{424} \mathrm{H}_{473} \mathrm{O}_{28} \mathrm{~N}_{58}$ 1365.8 , found 1635.7573 .

\section{References}

[1] (a) Wolfbeis, O. S. Adv. Mater. 2008, 20, 3759; (b) Mesli, A.; Dobaczewski, L.; Nielsen, K. B.; Kolkovsky, V.; Petersen, M. C.; Larsen, A. N. Phys. Rev. B 2008, 78, 165202; (c) Uchiyama, S.; de Silva, A. P.; Iwai, K. J. Chem. Educ. 2006, 83, 720; (d) Kucsko, G.; Maurer, P. C.; Yao, N. Y.; Kubo, M.; Noh, H. J.; Lo, P. K.; Park, H.; Lukin, M. D. Nature 2013, 500, 54-U71.
[2] (a) Childs, P. R. N.; Greenwood, J. R.; Long, C. A. Rev. Sci. Instrum. 2000, 71, 2959; (b) Seyedyagoobi, J. Rev. Sci. Instrum. 1991, 62, 249.

[3] (a) Lee, T. W.; Hegde, N. Combust. Flame 2005, 142, 314; (b) Chung, K.; Cho, J. K.; Park, E. S.; Breedveld, V.; Lu, H. Anal. Chem. 2009, 81, 991.

[4] (a) Ring, E. F. J. Infrared Phys. Techn. 2007, 49, 297; (b) Grattan, K T. V.; Palmer, A. W. Rev. Sci. Instrum. 1985, 56, 1784; (c) Dabiri, D. Exp. Fluids 2009, 46, 191.

[5] (a) Wang, X. D.; Wolfbeis, O. S.; Meier, R. J. Chem. Soc. Rev. 2013, 42, 7834; (b) Brites, C. D. S.; Lima, P. P.; Silva, N. J. O.; Millan, A.; Amaral, V. S.; Palacio, F.; Carlos, L. D. Nanoscale 2012, 4, 4799; (c) Song, Q. S.; Yang, S. S.; Sheng, R.; Li, T. Acta Chim. Sinica 2014, 72, 89 (in Chinese). (宋秋生, 杨森森, 盛锐, 李谭, 化学学 报, 2014, 72, 89.)

[6] (a) Liu, J.; Guo, X. D.; Hu, R.; Xu, J.; Wang, S. Q.; Li, S. Y.; Li, Y.; Yang, G. Q. Anal. Chem. 2015, 87, 3694; (b) Liu, X.; Li, S. Y.; Feng, J.; Li, Y.; Yang, G. Q. Chem. Commun. 2014, 50, 2778; (c) Okabe, K.; Inada, N.; Gota, C.; Harada, Y.; Funatsu, T.; Uchiyama, S. Nat. Commun. 2012, 3, 705; (d) Feng, J.; Tian, K. J.; Hu, D. H.; Wang, S. Q.; Li, S. Y.; Zeng, Y.; Li, Y.; Yang, G. Q. Angew. Chem.-Int. Ed. 2011, 50, 8072; (e) Feng, J.; Xiong, L.; Wang, S. Q.; Li, S. Y.; Li, Y.; Yang, G. Q. Adv. Funct. Mater. 2013, 23, 340; (f) Ebrahimi, S. Akhlaghi, Y.; Kompany-Zareh, M.; Rinnan, A. ACS Nano 2014, 8 , 10372; (g) Zhegalova, N. G.; Dergunov, S. A.; Wang, S. T.; Pinkhassik, E.; Berezin, M. Y. Chem.-Eur. J. 2014, 20, 10292; (h) Hu, X. L.; Li, Y.; Liu, T.; Zhang, G. Y.; Liu, S. Y. ACS Appl. Mater. Interfaces 2015, 7, 15551; (i) Song, Q. S.; Zhou, W.; Wu, X. M.; Wu, F. Acta Chim. Sinica 2016, 74, 435 (in Chinese). (宋秋生, 周 稳, 吴新民, 吴凡, 化学学报, 2016, 74, 435.)

[7] Ross, D.; Gaitan, M.; Locascio, L. E. Anal. Chem. 2001, 73, 4117.

[8] Ye, F.; Wu, C.; Jin, Y.; Chan, Y.-H.; Zhang, X.; Chiu, D. T. J. Am. Chem. Soc. 2011, 133, 8146.

[9] Ozawa, A.; Shimizu, A.; Nishiyabu, R.; Kubo, Y. Chem. Commun. 2015, 51,118 .

[10] (a) Kojima, C.; Irie, K.; Tada, T.; Tanaka, N. Biopolymers 2014, 101, 603; (b) Cakara, D.; Kleimann, J.; Borkovec, M. Macromolecules 2003, 36, 4201.

[11] (a) Zeng, Y.; Li, Y. Y.; Li, M.; Yang, G. Q.; Li, Y. J. Am. Chem. Soc. 2009, 131, 9100; (b) Zhang, X. H.; Zeng, Y.; Yu, T. J.; Chen, J. P.; Yang, G. Q.; Li, Y. Langmuir 2014, 30, 718; (c) Liu, X. Y.; Zeng, Y.; Liu, J.; Li, P.; Zhang, D. S.; Zhang, X. H.; Yu, T. J.; Chen, J. P.; Yang, G. Q.; Li, Y. Langmuir 2015, 31, 4386; (d) Li, P.; Zeng, Y.; Chen, J. P.; Li, Y. Y.; Li, Y. Acta Chim. Sinica 2012, 70, 1611 (in Chinese). (李鹏，曾毅，陈金平，李迎迎，李嫕，化学学报，2012， 70, 1611.)

[12] Zeng, Y.; Li, Y. Y.; Yuan, Z.; Li, Y. Acta Chim. Sinica 2009, 67, 2714 (in Chinese). (曾毅, 李迎迎, 袁钊, 李嫕，化学学报，2009，67, 2714.)

[13] (a) Baker, G. A.; Baker, S. N.; McCleskey, T. M. Chem. Commun. 2003, 2932; (b) Lou, J. F.; Hatton, T. A.; Laibinis, P. E. Anal. Chem. 1997, 69, 1262.

[14] Sehgal, R. K.; Kumar, S. Org. Prep. Proced. Int. 1989, 21, 223.

(Cheng, B.) 